\title{
Strategic knowledge maps of the techno-scientific network (SK maps)
}

1. José Pino-Díaz ${ }^{1}$,

2. Evaristo Jiménez-Contreras ${ }^{2}$,

3. Rosario Ruíz-Baños ${ }^{3}$,

4. Rafael Bailón-Moreno ${ }^{4}$

Article first published online: 30 MAR 2012

DOI: $10.1002 /$ asi.21712

(C) 2011 ASIS\&T

Issue

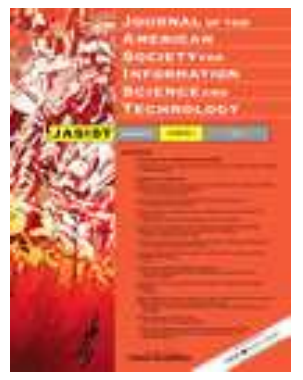

\section{Journal of the American Society for Information Science and Technology}

Volume 63, Issue 4, (/doi/10.1002/asi.v63.4/issuetoc) pages 796-804, April 2012

Additional Information

\section{How to Cite}

Pino-Díaz, J., Jiménez-Contreras, E., Rúz-Baños, R. and Bailón-Moreno, R. (2012), Strategic knowledge maps of the techno-scientific network (SK maps). J. Am. Soc. Inf. Sci., 63: 796-804. doi: 10.1002/asi.21712

\section{Author Information}

1 Departamento de Filología Griega, Estudios Árabes, Lingüística General y Documentación, Universidad de Málaga, Málaga, Spain 
2 Departamento de Biblioteconomía y Documentación, Universidad de Granada, Granada, Spain

3 Departamento de Biblioteconomía y Documentación, Universidad de Granada, Granada, Spain

4 Departamento de Ingeniería Química, Universidad de Granada, Granada, Spain

Email: José Pino-Díaz (jpinod@uma.es), Evaristo Jiménez-Contreras (evaristo@ugr.es), Rosario Ruíz-Baños (rruizb@ugr.es), Rafael Bailón-Moreno (bailonm@ugr.es)

\section{Publication History}

1. Issue published online: 30 MAR 2012

2. Article first published online: 30 MAR 2012

3. Manuscript Accepted: 26 OCT 2011

4. Manuscript Revised: 25 OCT 2011

5. Manuscript Received: 3 NOV 2010

- Abstract

- Article (/doi/10.1002/asi.21712/full)

- References (/doi/10.1002/asi.21712/references)

- Cited By (/doi/10.1002/asi.21712/citedby)

View Full Article (HTML) (/doi/10.1002/asi.21712/full) Get PDF (968K) (/doi/10.1002/asi.21712/pdf) Keywords:

competitive intelligence; maps (graphic representation); decision making

Knowledge engineering and information mapping are two recent scientific disciplines in constant development where mathematics, linguistics, computer science, and information visualization converge. Their main focus is to discover and display new knowledge in large document databases. They have broad and innovative fields of application for strategic scouting in science and technology, knowledge management, business intelligence, and scientific and technological evaluation. This article presents a new method for mapping the strategic research network and illustrates its application to the strategic analysis of the knowledge domain "Spanish Research in Protected Areas for the Period 1981-2005." This strategic knowledge is displayed through a set of two-dimensional cartographic maps and three-dimensional images of two networks: the international network WoS_KWAJ (1981-2005) and the national network IEDCYT_KWAJ (1981-2005). These maps can be very useful in decision-making processes for science and technology policy.

View Full Article (HTML) (/doi/10.1002/asi.21712/full) Get PDF (968K) (/doi/10.1002/asi.21712/pdf)

\section{More content like this}

Find more content:

- like this article (/advanced/search/results? articleDoi $=10.1002 /$ asi.21712\&scope $=$ allContent $\&$ start $=1 \&$ resultsPerPage $=20$ )

Find more content written by: 
- José Pino-Díaz (/advanced/search/results?searchRowCriteria[0].queryString="Jos\%C3\%A9 Pino$\mathrm{D} \% \mathrm{C} 3 \% \mathrm{ADaz} " \&$ searchRowCriteria[0].fieldName=author\&start=1\&resultsPerPage $=20$ )

- Evaristo Jiménez-Contreras (/advanced/search/results?searchRowCriteria[0].queryString="Evaristo $\underline{\mathrm{Jim} \% \mathrm{C} 3 \% \mathrm{~A} 9 \text { nez-Contreras"\&searchRowCriteria[0].fieldName=author\&start=1\&resultsPerPage=20) }}$

- Rosario Ruúz-Baños (/advanced/search/results?searchRowCriteria[0].queryString="Rosario Ru\%C3\%ADzBa\%C3\%B1os"\&searchRowCriteria[0].fieldName=author\&start=1\&resultsPerPage $=20$ )

- Rafael Bailón-Moreno (/advanced/search/results?searchRowCriteria[0].queryString="Rafael Bail\%C3\%B3n-Moreno"\&searchRowCriteria[0].fieldName=author\&start=1\&resultsPerPage=20)

- All Authors (/advanced/search/results?searchRowCriteria[0].queryString="Jos\%C3\%A9 PinoD\%C3\%ADaz" "Evaristo Jim\%C3\%A9nez-Contreras" "Rosario Ru\%C3\%ADz-Ba\%C3\%B1os" "Rafael Bail\%C3\%B3n-Moreno"\&searchRowCriteria[0].fieldName=author\&start=1\&resultsPerPage=20) 